\title{
Cardiac torsion after excision of a huge mature cystic
} \section{teratoma}

\section{Kathleen S Cruz* and Jaime F Esquivel}

TCVS subspecialty resident, UP-Philippine General Hospital, Philippines

\section{Introduction}

Cardiac torsion is a rare complication following pericardiectomy, but has a high mortality rate, reported at 30 to $64 \%$ in some case reports [1]. Cardiac herniation has been noted in some literature after a pneumonectomy with partial pericardiectomy [2] and excision of pericardial thymic tumor [3]. In other cases, it was found only at autopsy. There are no case reports on cardiac torsion after excision of a teratoma.

\section{Case report}

This is the case of J.F., a 52-year-old female with a 12 -year history of a progressively enlarging mediastinal cyst. She came to the clinic with occasional shortness of breath, especially during exertion. She had no other comorbidities. She had a previous computed tomography scan of the chest done 12 years prior to consult, which revealed a large hypodense, non-enhancing, right middle mediastinal mass with a calcified, thick-walled capsule measuring $1.6 \times 1.3 \times 1.2 \mathrm{~cm}$. The last scan made four months prior to admission showed a $15 \times 10 \times 20$ $\mathrm{cm}$ mediastinal cyst displacing the trachea and the heart to the left hemithorax (Figure 1). Preoperative chest radiograph showed a huge, round opacity in the right chest with mediastinal shift (Figure 2). Echocardiography showed normal cardiac function.

The intial diagnosis was a pericardial cyst. She underwent an initial attempt for a video-assisted thoracoscopic surgery with aspiration of the cyst. However, thick adhesions around the cyst were noted during thoracoscopic evaluation of the chest. A median sternotomy was performed with excision of the cyst and en bloc partial pericardiectomy. Intraoperatively, the cyst was $20 \times 20 \mathrm{~cm}$ with a thick, calcified wall and adherent to, but not invading, the middle and lower lung lobes and the diaphragm (Figure 3). The middle and lower lobes were collapsed and

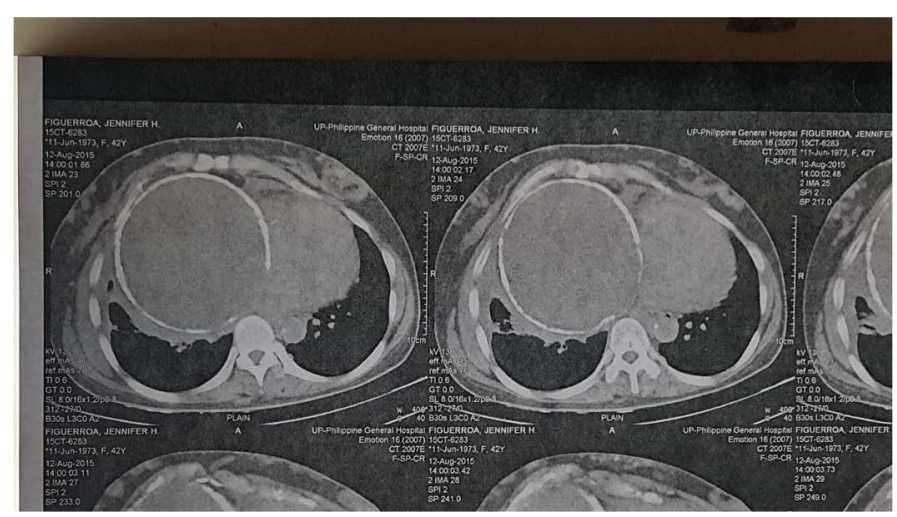

Figure 1. Chest computed tomography scan showing cystic mass at the right hemithorax (arrow). non-expanding, probably from chronic compression by the mass. The pericardial defect was approximately $10 \times 10 \mathrm{~cm}$. No reconstruction of the pericardium was done. The patient was extubated postoperatively. She started having episodes of hypotension attributed to hypovolemia; hence, blood transfusion was initiated. Immediate postoperative hemoglobin was $127 \mathrm{~g} / \mathrm{L}$, which dropped to $107 \mathrm{~g} / \mathrm{L}$ on repeat. Blood pressure was normalized after transfusion and fluid replacement. Chest radiograph was done immediately after the operation, which showed a lower lobe opacity on the right (Figure 4). The lower lobe opacity

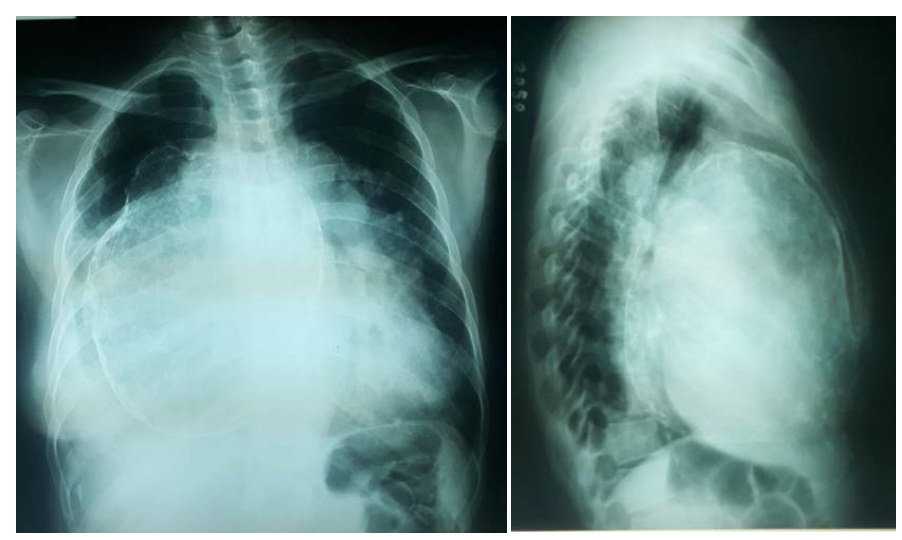

Figure 2. Preoperative chest radiograph posteroanterior and lateral views.

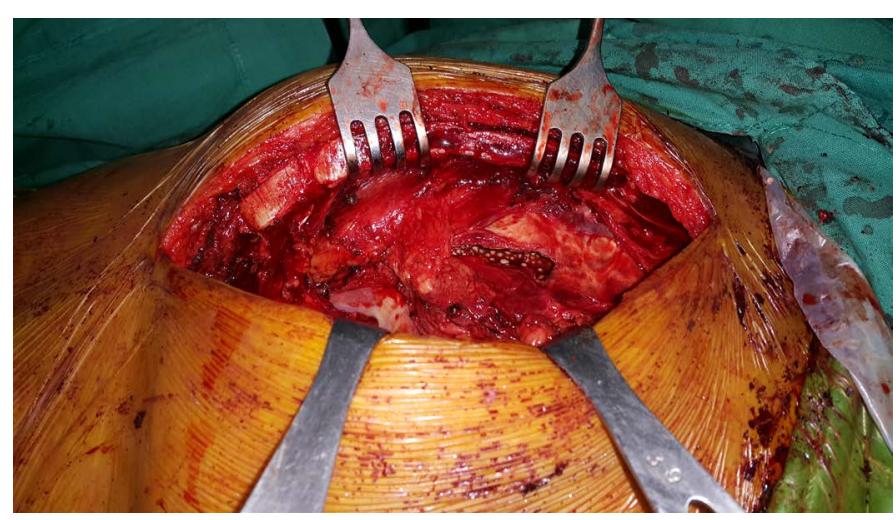

Figure 3. Intraoperative view of the mass through the sternotomy incision.

Correspondence to: Kathleen Cruz. MD, TCVS subspecialty resident, UPPhilippine General Hospital E-mail: kathcruz@gmail.com

Received: August 03, 2016; Accepted: August 21, 2016; Published: August 24, 2016 


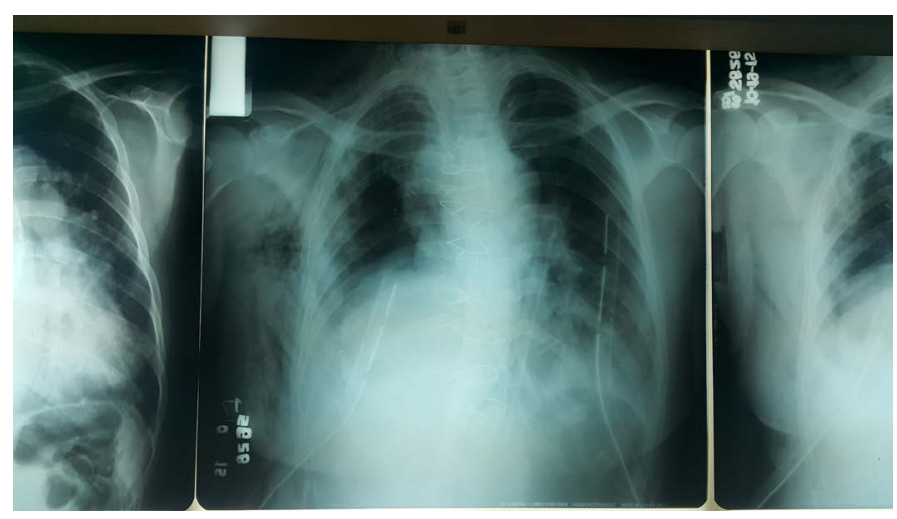

Figure 4. Immediate postoperative chest X-ray.

A

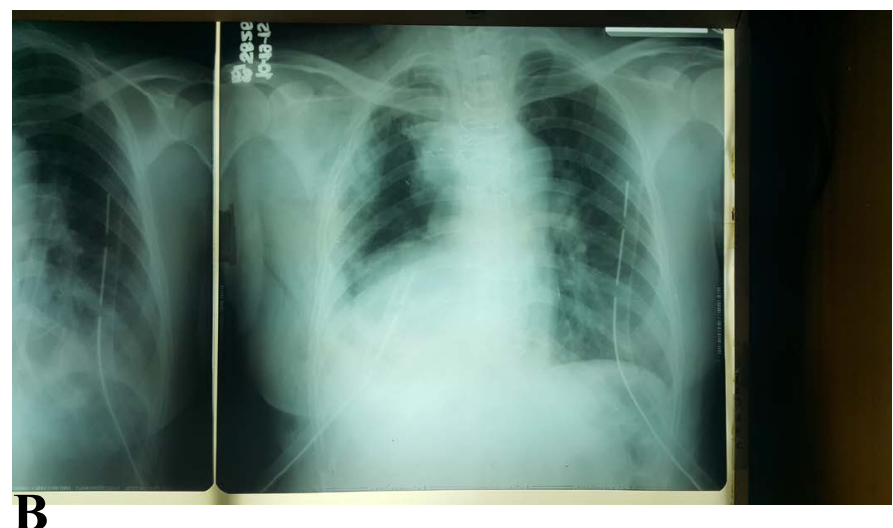

B

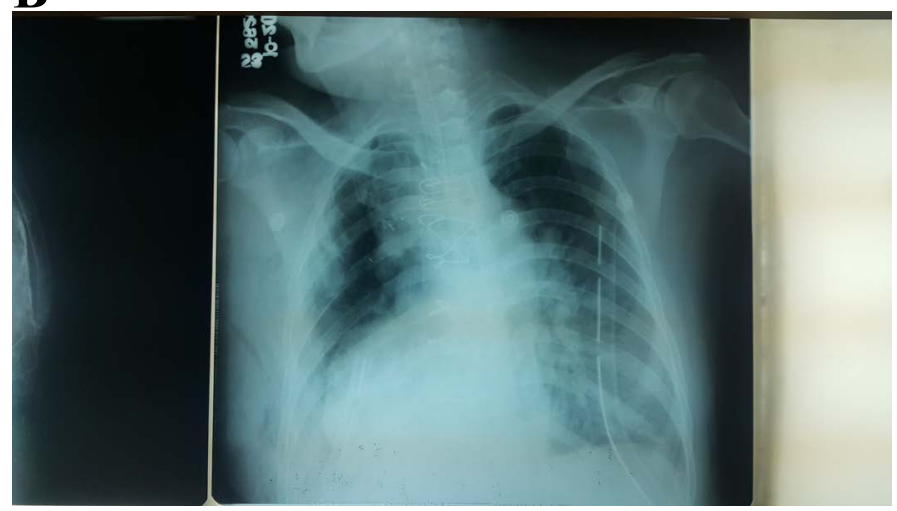

Figure 5 [A\&B]. Repeat chest radiograph day 3 (A) and postoperative day 4 (B).

was attributed to atelectasis. Patient was started on aggressive chest physiotherapy and spirometry. Patient was feeding well and had no episode of dyspnea. Two days after the operation, patient developed sensorial changes with shallow breathing. Arterial blood gas showed respiratory acidosis, with $\mathrm{pH} 7.233, \mathrm{pCO} 272.3 \mathrm{mmHg}$ and $\mathrm{pO} 2$ $131.6 \mathrm{mmHg}$ on $\mathrm{FiO} 240 \%$ via a face mask. Oxygen saturation was maintained at $99 \%$. Electrocardiogram showed sinus tachycardia. She was intubated and admitted to the intensive care unit. On physical examination, she had an edematous face, upper trunk and bilateral upper extremity consistent with a superior vena cava obstruction. Initial impression was mediastinal shifting due to the negative pressure on the right hemithorax (Figures 5A and 5B). Chest tube was clamped and repositioning of the patient was attempted with no significant change in the chest radiograph. On the third postoperative day, she became hypotensive requiring inotropic support. Hypotension was persistent until the $4^{\text {th }}$ postoperative day, despite fluid resuscitation and with maximum inotropic requirements. Repeat chest radiograph revealed the apex of the heart located at the right chest. ECG showed sinus tachycardia with occasional premature ventricular contractions. Confirmatory echocardiography showed cardiac torsion. She was immediately brought to the operating room for emergency sternotomy and repositioning of the heart.

Intraoperatively, the heart herniated out of the pericardial space. The cardiac apex was shifted to the right. Repositoning was done and the pericardium was reconstructed using a polytetrafluoroethylene(PTFE) felt patch to hold the heart in place. Blood pressure increased from 70 palpatory to a systolic blood pressure of $120 \mathrm{mmHg}$, with decreasing inotropic support. The patient, however, arrested during sternal closure and succumbed to severe metabolic acidosis. Histopathology revealed mature cystic teratoma (Figures $6 \mathrm{~A}$ and $6 \mathrm{~B}$ ).

\section{Discussion}

Cardiac herniation occurs most commonly after intrapericardial pneumonectomy. There have been reports of herniation after residual pericardial defects or wide excision of pericardium after surgery for thymic tumors. It usually manifests within 24 hours postoperatively. Findings of sudden hypotension, signs of superior vena cava obstruction such as facial and upper extremity edema and arrhythmias have been reported. Often than not, surgeons misdiagnose this as acute hemorrhage or acute myocardial infarction [2].

Mortality rate is high at $50 \%$ [3]. The hemodynamics of cardiac torsion result from compression of the atriocaval junctions and great vessels as the heart is rotated to the right side, leading to decreased

\section{A}

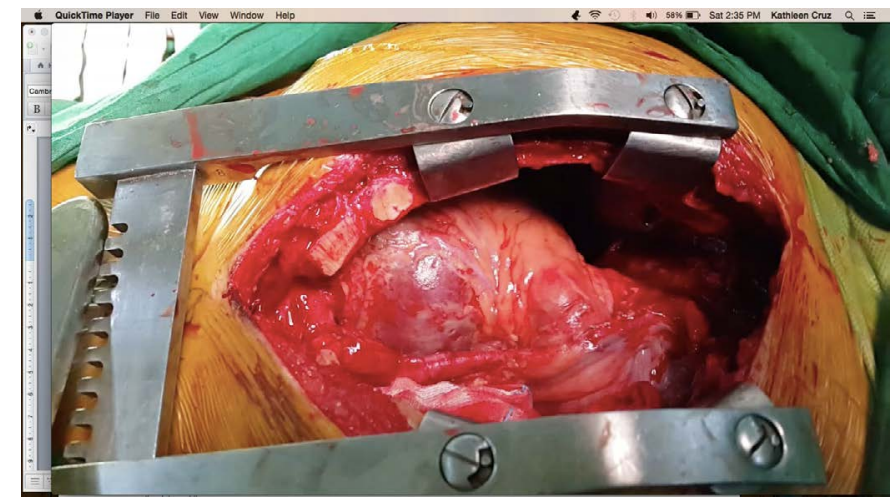

B

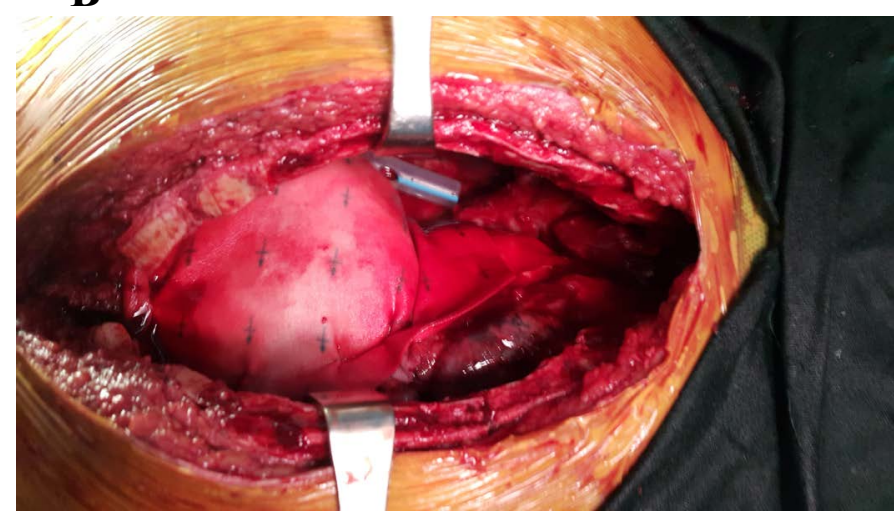

Figure 6 [A \& B]. Cardiac torsion (A). Heart repositioned and pericardial patching done with felt (B). 
venous return and hence, decreased cardiac output [4,5]. Left-sided cardiac herniation has a different mechanism. Strangulation of the ventricular muscles and pressure over the coronary arteries by the edges of the pericardial defect causes the hemodynamic compromise [2].

Conservative measures have been proposed such as repositioning the patient or injection of air into the surgical hemithorax. Effectiveness of such strategies, however, are still undetermined [4]. In our patient, repositioning of the patient and clamping of the right chest tube to induce positive pressure in the surgical hemithorax did not improve or reverse the patient's condition. Surgical repositioning with reconstruction of the pericardium corrected the torsion. If recognized and managed early, the hemodynamic consequences of cardiac torsion can be reversed.

\section{Conclusion}

Cardiac herniation and torsion should be anticipated in patients with a wide pericardial defect and non-expanded or absent lung on the side of the defect. Given the high mortality rate of late recognition of such disease entity, this author recommends reconstruction of the pericardium after excision of a mediastinal tumor with a wide en bloc resection of the right or left side of the pericardium and a nonexpanding lung.

\section{References}

1. Onem G, Baltalarli A, Sungurtekin H, Evrengul H, Ozcan AV, et al. (2006) Iatrogenic cardiac herniation and torsion after surgery for a penetrating cardiac injury. Tex Heart Inst J 33: 526-528. [Crossref]

2. Shrinivas G (2010) Cardiac herniation following completion pneumonectomy for bronchiectasis. Ann Cardiac Anes 13: 249-251.

3. Numata S, Aye WM, Lee CN (2005) Cardiac herniation after resection of pericardial thymic cyst. Interact Cardiovasc Thorac Surg 4: 350-351. [Crossref]

4. Cassorla L, Katz JA (1984) Management of cardiac herniation after intrapericardial pneumonectomy. Anesthesiology 60: 362-364. [Crossref]

5. Kawamukai K, Antonacci F, Di Saverio S, Boaron M (2011) Acute postoperative cardiac herniation. Interact Cardiovasc Thorac Surg 12: 73-74. [Crossref]

Copyright: (C2016 Cruz KS. This is an open-access article distributed under the terms of the Creative Commons Attribution License, which permits unrestricted use, distribution, and reproduction in any medium, provided the original author and source are credited. 